\title{
ARTí́culo
}

\section{Relación del crecimiento, condición y supervivencia del ostión del Pacífico Crassostrea gigas y las variables ambientales, cultivado en suspensión en el sistema lagunar Navachiste-Macapule, Sinaloa, México}

Growth, condition and survival relationships of the Pacific oyster Crassostrea gigas and environmental variables, cultured in suspension in the Navachiste-Macapule lagoon system, Sinaloa, Mexico

\author{
Gerardo Rodríguez-Quiroz ${ }^{1}$, Manuel García-Ulloa ${ }^{1}$, Ana Laura \\ Domínguez-Orozco ${ }^{1}$, Teresa Natividad Valenzuela-Hernández ${ }^{1}$, \\ Eusebio Nava-Pérez ${ }^{1}$ y Andrés Martín Góngora-Gómez ${ }^{1 *}$
}

\begin{abstract}
${ }^{1}$ Instituto Politécnico Nacional (IPN), Centro Interdisciplinario de Investigación para el Desarrollo Integral Regional, Unidad Sinaloa (CIIDIR-Sinaloa), Bulevar Juan de Dios Bátiz Paredes \#250, Colonia San Joachin, Guasave, Sinaloa, 81101, México. *gogam69@hotmail.com
\end{abstract}

\begin{abstract}
Evaluation of potential culture sites represents a 'good practice' alternative to support the development of oyster industry at the northeast of Mexico. The relationship of growth, condition and survival of Crassostrea gigas with the environmental parameters was studied at the Navachiste-Macapule lagoon system (Sinaloa, Mexico) from November 2011 to November 2012. The culture was carried out using 2,520 small oysters ( $3.5 \pm 0.5 \mathrm{~mm}$ shell height) in a suspended longline system. The environmental parameters and oyster biometrics were sampled each 15 days. The mean values were: temperature $=25.01 \pm 5.29^{\circ} \mathrm{C}$, dissolved oxygen $=6.10 \pm 1.93 \mathrm{mg} \mathrm{L}^{-1}$, salinity $=33.5, \mathrm{pH}=7.82 \pm 0.39$ and depth $=1.66 \pm 0.55 \mathrm{~m}$. The concentrations of total suspended solids, particulate organic matter and chlorophyll a fluctuated from 41.94 to 125.22 $\mathrm{mg} \mathrm{L}^{-1}, 8.91$ to $15.22 \mathrm{mg} \mathrm{L}^{-1}$, and 13.36 to $3.69 \mathrm{mg} \mathrm{m}^{-3}$, respectively. After 13 culture months, the shell height reached $95.05 \pm$ $9.21 \mathrm{~mm}$, length $85.64 \pm 20.01 \mathrm{~mm}$, width $28.76 \pm 10.91 \mathrm{~mm}$, and the total weight $83.58 \pm 17.18 \mathrm{~g}$. The daily growth rate for the shell height and total weight were $0.25 \mathrm{~mm} \mathrm{day}^{-1}$ and $0.23 \mathrm{~g} \mathrm{day}^{-1}$, respectively. Significant differences were found for the total weight and the shell metric values throughout the culture. The final survival and mean condition index were $94.72 \%$ and $34.65 \pm 4.46$, respectively. Water temperature exerted a positive stronger effect on shell height and total weight. Results show that $\mathrm{C}$. gigas reached the commercial size $(80 \mathrm{~mm} \mathrm{SH})$ after 8 months of culture under the environmental conditions of the Navachiste-Macapule lagoon system, Sinaloa, Mexico. Obtained data contribute with basic information on further studies and commercial production cycles of this species in the region.
\end{abstract}

Key words: Culture, suspended plastic trays, growth rate, Crassostrea gigas, mortality, Mexico

\begin{abstract}
Resumen.- La evaluación de nuevos lugares con potencial de cultivo en la industria ostrícola representa una 'buena práctica' que contribuye con el desarrollo de esta actividad en el noroeste de México. Se realizó un cultivo en suspensión (long-line) con el ostión Crassostrea gigas para estudiar la relación de su crecimiento, condición y supervivencia con las variables ambientales en el sistema lagunar Navachiste-Macapule (Sinaloa, México), de noviembre 2011 a noviembre 2012. Se utilizaron 2.520 semillas $(3,5 \pm 0,5 \mathrm{~mm}$ de altura de valvas). Las variables físicas y químicas del agua, y biométricas del ostión fueron registradas cada 15 días obteniendo los siguientes valores promedio: temperatura $=25,01 \pm 5,29^{\circ} \mathrm{C}$; oxígeno disuelto $=6,10 \pm 1,93 \mathrm{mg} \mathrm{L}^{-1}$; salinidad $=32,88 \pm 2.15 ; \mathrm{pH}=7,82 \pm 0,39$ y profundidad $=1,66 \pm 0,55 \mathrm{~m}$. Las concentraciones de sólidos suspendidos totales, materia orgánica particulada y clorofila a variaron de 41,94 a $125,22 \mathrm{mg} \mathrm{L}^{-1} ; 8,91$ a 15,22 $\mathrm{mg} \mathrm{L}^{-1}$ y 13,36 a 3,69 $\mathrm{mg} \mathrm{m}^{-3}$, respectivamente. La altura de la concha alcanzó 95,05 $\pm 9,21 \mathrm{~mm}$; la longitud 85,64 $\pm 20,01 \mathrm{~mm}$; el ancho $28,6 \pm 10,91 \mathrm{~mm}$ y el peso $83,58 \pm 17,18 \mathrm{~g}$. La tasa de crecimiento diaria del ostión fue de $0,25 \mathrm{~mm} \mathrm{día}^{-1} \mathrm{para}$ la altura de la concha y de $0,23 \mathrm{~g} \mathrm{día}^{-1}$ para el peso. Se encontraron diferencias significativas en el peso total y en los valores métricos de las conchas. La supervivencia final fue de 94,72\%, y el índice de condición promedio de 34,65 $\pm 4,46$. La temperatura del agua ejerció un fuerte efecto positivo en la altura de las valvas y el peso total del ostión. Los resultados muestran que C. gigas alcanzó la talla comercial $(80 \mathrm{~mm}$ de altura de las valvas) después de 8 meses de cultivo bajo las condiciones ambientales del sistema lagunar Navachiste-Macapule, Sinaloa, México. Los datos obtenidos contribuyen con información básica necesaria para futuros estudios y ciclos de producción comercial de esta especie en la región.
\end{abstract}

Palabras clave: Cultivo, canastas de plástico suspendidas, tasa de crecimiento, Crassostrea gigas, mortalidad, México 


\section{INTRODUCCIÓN}

El ostión del Pacífico Crassostrea gigas representa la especie de molusco más cultivada en el mundo. La estadística global para este bivalvo en 2010 arrojó una producción aproximada de 653,000 ton (Helm 2015). Sin embargo, desde hace varias décadas, se han documentado eventos de mortalidad masiva en diferentes países (Glude 1974, Soletchnick et al. 1999, Cheney et al. 2000) que afectan la industria acuícola de tal manera, que la producción total en 2013 disminuyó más de $15 \%$, lo cual fue equivalente a un volumen de cosecha de 556,000 ton (Helm 2015). C. gigas fue introducido en la década de los años '70 en varios estados de México (Baja California, Sonora, Sinaloa y Nayarit) y cultivado con éxito hasta finales de los años ' 90 . Debido a la poca inversión en la industria y a causas de mortalidad desconocidas, entre algunos factores, la actividad ostrícola de México sufrió un proceso de estancamiento a partir de 1997. Posteriormente, se detectaron agentes causales de la mortalidad como el herpesvirus y el protozoario Perkinsus sp., pero a partir del 2010 la producción ostrícola continúa presentando signos de recuperación (ChávezVillalba et al. 2010, Chávez-Villalba 2014). Las mortalidades presentadas se atribuyen principalmente a una afección en los ostiones conocida como 'síndrome de mortalidad de verano' cuyo origen permanece aún desconocido. Algunas teorías basadas en la combinación e interacción de factores extrínsecos (bióticos y abióticos) e intrínsecos (genéticos, inmunológicos y fisiológicos), son usadas frecuentemente para explicar la drástica reducción de bivalvos en cultivo (Royer et al. 2007, EnríquezEspinoza et al. 2010). Derivado de esfuerzos coordinados del gobierno con los productores y centros de investigación para tratar de conocer las causas de mortalidad en C. gigas, se ha concluido que la presencia de patógenos (Enríquez-Espinoza et al. 2010, Vázquez-Yeomans et al. 2010, Enríquez-Espinoza et al. 2015) y/o la combinación de parámetros ambientales (Chávez-Villalba et al. 2007, Castillo-Durán et al. 2010) pueden estar asociados a la reducción del cultivo. No obstante, la erradicación de los patógenos y el control de las condiciones del medio ambiente son tareas imposibles, por lo que la única opción para los productores parece ser el seguimiento de buenas prácticas de cultivo para minimizar el impacto de las mortalidades (Enríquez-Espinoza et al. 2010). El cultivo de ostiones a baja densidad (Grabowski et al. 2004), fuera de aguas protegidas (Chávez-Villalba et al. 2010) y en determinadas estaciones del año (Castillo-Durán et al. 2010) son estrategias asociadas a buenas prácticas de producción en C. gigas, que han sido utilizadas para disminuir el impacto de mortalidades en la industria ostrícola. La evaluación de nuevos sitios de cultivo representa otra alternativa, ya que las condiciones ambientales de cada localidad pueden influir en la respuesta de crecimiento general de los moluscos. La temperatura de agua (Ren et al. 2000) y la concentración de clorofila $a$ (Ulloa et al. 2003) representan los factores de mayor influencia en la fisiología y crecimiento del ostión, entre otros muchos como la salinidad, oxígeno disuelto y seston (ChávezVillalba et al. 2007). Aún más, diferentes localidades con distintas condiciones dentro de un mismo cuerpo de agua pueden afectar el desempeño de los animales. Por ejemplo, García-Ulloa et al. (2006) obtuvieron diferentes tasas de crecimiento del ostión japonés cultivado en un estero ubicado en la costa media del Pacífico mexicano, a distintas profundidades, velocidad de las corrientes y sustratos.

La mayoría de los trabajos relacionados con la 'mortalidad de verano' en ostión y su posible mitigación, han sido realizados en los estados de Baja California y Sonora (Cáceres-Martínez et al. 2004, Chávez-Villalba et al. 2005, 2007; MaedaMartínez 2008, Castillo-Durán et al. 2010), pero debido a su frontera hacia el sur y la posible similitud de condiciones ambientales, las granjas ostrícolas del estado de Sinaloa pudieran ser afectadas. No existen reportes de mortalidad de C. gigas en granjas de Sinaloa hasta la fecha, pero sí acerca de la presencia de los mismos parásitos (Villanueva-Fonseca $\&$ Escobedo-Bonilla 2013) con aquellos reportados en Sonora y Baja California (Enríquez-Espinoza et al. 2010, 2015). En el caso de la influencia de los factores del medio ambiente, Góngora-Gómez et al. (2012) evaluaron el crecimiento de $C$. gigas en el Estero La Piedra, al norte de Sinaloa, obteniendo organismos de talla comercial en 7 meses de cultivo (temporada otoño-primavera) y una supervivencia de $88 \%$. Con tal estrategia, la temporada de verano, asociada a las mortalidades, puede ser evitada. El objetivo de este trabajo fue evaluar el crecimiento, índice de condición y supervivencia de C. gigas durante el periodo de noviembre 2011 a noviembre 2012, así como su relación con las variables fisicoquímicas, en el sistema lagunar Navachiste-Macapule, a fin de aportar información para su manejo acuícola en la región.

\section{Materiales Y MÉTODOS}

El sistema lagunar Navachiste-Macapule $\left(25^{\circ} 21^{\prime} \mathrm{N}\right.$ y $108^{\circ} 30^{`} \mathrm{~W}$ ) está localizado a lo largo de la costa noroeste del estado de Sinaloa (Fig. 1). Se utilizaron 2.520 juveniles (3,5 \pm $0,5 \mathrm{~mm}$ ) adquiridos de un criadero comercial (Acuacultura Robles S. P, R. de R. I., Sonora, México) los cuales fueron transportados hasta el sitio de cultivo a baja temperatura $\left(10^{\circ} \mathrm{C}\right)$. Antes de ser introducidos al estero se revisó una muestra al microscopio para verificar su tamaño, color y viabilidad mediante la detección de movimiento dentro de la concha. 


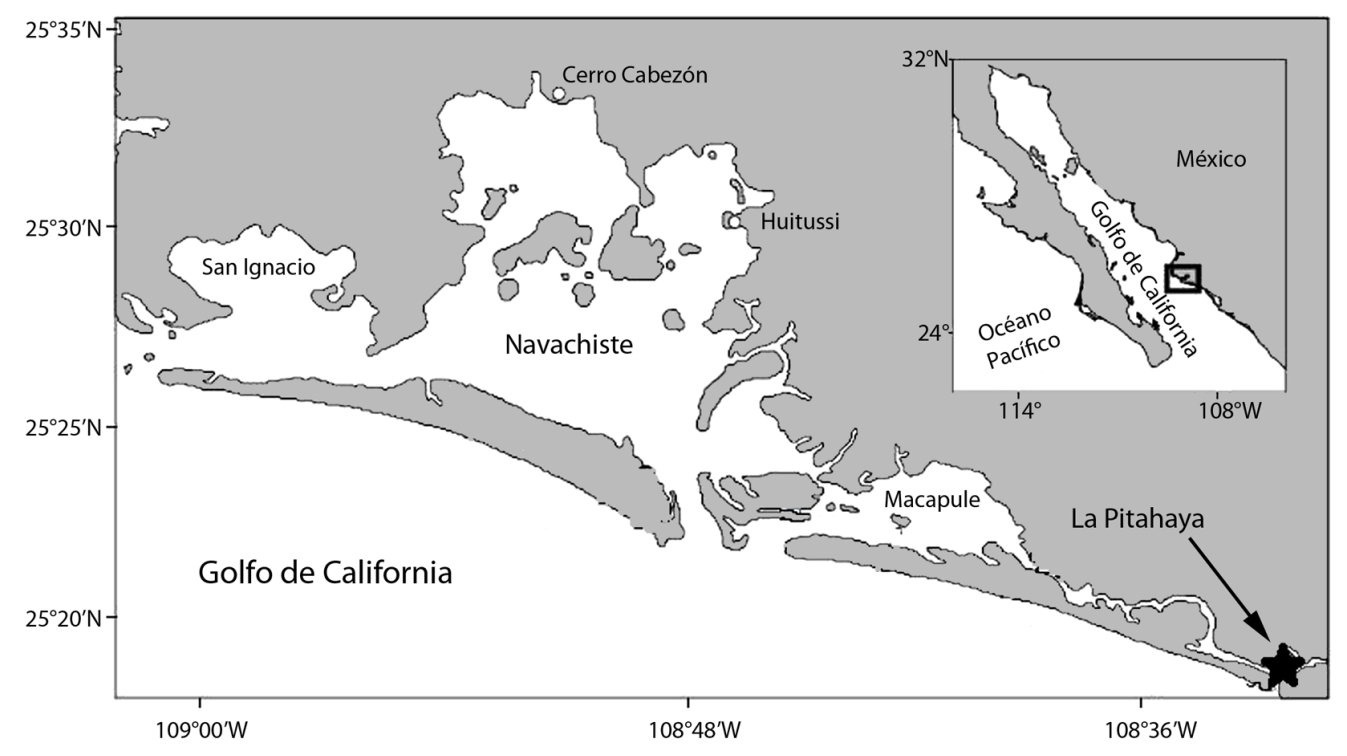

Figura 1. Localización geográfica del sitio de cultivo del ostión japonés Crassostrea gigas en el sistema lagunar Navachiste-Macapule, Sinaloa, México / Geographical location of the culture site for the Japanese oyster, Crassostrea gigas, in the Navachiste-Macapule lagoon system, Sinaloa, Mexico

Después, se aclimataron (Gallo-García et al. 2001) y dividieron en lotes de aproximadamente 250 animales que fueron embolsados en tela mosquitera $(2 \mathrm{~mm})$ de $20 \times 20 \mathrm{~cm}$. Los ostiones fueron cultivados de acuerdo a la forma comercial vigente de los granjeros, con una fase inicial de crecimiento que consta del mantenimiento de los organismos en bolsas mosquiteras dentro de canastas ostrícolas $\left(\mathrm{Nestier}^{\mathrm{TM}}\right)$ durante los dos primeros meses, y la fase terminal de engorda consistente en su crecimiento durante 11 meses fuera de las bolsas mosquitero pero dentro de las canastas. Para ambas fases, las canastas permanecen atadas a una línea madre manteniendo en suspensión el cultivo. La fase inicial comenzó en noviembre 2011 y la población de ostiones fue desdoblada 4 veces (cada 15 días) hasta que los organismos alcanzaron una altura de la concha mayor de $30 \mathrm{~mm}$, la cual representa el tamaño necesario para que los ostiones no pasen por los orificios y sean retenidos en las canastas. En el desdoble, los organismos de cada bolsa son vertidos sobre la canasta bajo la cual se coloca un recipiente plástico con agua marina. De esta forma, la canasta sirve como tamiz para seleccionarlos por tamaño. Los ostiones que pasaran por los orificios de las canastas fueron devueltos a la bolsa mosquitero, mientras que los que son retenidos se sembraron a una densidad de 42 ostiones por canasta. Según GóngoraGómez et al. (2012), cada módulo se compone de 7 canastas: a la primera o superior se le colocan flotadores plásticos, las 5 siguientes contienen los ostiones, y en la última o inferior se distribuyen piedras que sirven para mantener la forma vertical del módulo en suspensión. Se usan las canastas que sean necesarias para formar los módulos se acuerdo a la cantidad de ostiones cuya altura de la concha rebase $30 \mathrm{~mm}$ y a la supervivencia observada en cada desdoble. De esta manera, se ajustó una densidad de 42 ostiones por canasta hasta el final del estudio (noviembre 2012). La limpieza de las bolsas y canastas fue realizada cada 2 semanas; al mismo tiempo, los ostiones fueron limpiados con un cepillo de cerdas suaves, y tanto predadores (peces, caracoles y cangrejos) como competidores (filtradores como moluscos y pequeños invertebrados) eran separados de las canastas. Con el fin de estimar la mortalidad acumulada, las conchas vacías se recolectaron y contaron en cada limpieza (Góngora-Gómez et al. 2012).

Se obtuvieron los valores de temperatura del agua, oxígeno disuelto (OD, YSI, 55/12FT, Ohio 45387), salinidad (Atago, S/Mill), pH (Hanna, HI 8314) y transparencia (disco Secchi) cada 15 días, mientras que los sólidos suspendidos totales (SST) y la materia orgánica particulada (MOP) fueron medidos cada mes con el método gravimétrico (APHA 1995). La concentración de clorofila $a(\mathrm{Cl}-a)$ se obtuvo espectrofotométricamente utilizando el método descrito por Strickland \& Parsons (1972).

Después de cada limpieza, 50 ostiones seleccionados al azar eran medidos (altura, longitud y ancho de la concha, $\mathrm{mm}$ ) usando una regla vernier $( \pm 0,01 \mathrm{~mm})$ y pesados con una 
balanza de campo $( \pm 0,01 \mathrm{~g})$. Se obtuvieron la tasa de crecimiento diaria (longitud o peso final - longitud opeso inicial/ días de cultivo), y la tasa de crecimiento instantáneo relativo (TCIR) (Xiao et al. 2005), mediante la fórmula:

$$
\mathrm{TCIR}=\left(\operatorname{Ln} L_{2}-\operatorname{Ln} L_{1}\right) / \mathrm{t} \cdot 100
$$

donde $L_{1}$ y $L_{2}=$ longitud o peso final e inicial, respectivamente, $\mathrm{y} \mathrm{t}=$ tiempo de cultivo entre 2 muestreos. También, se obtuvieron la tasa de crecimiento absoluto (TCA), TCA $=\left(\mathrm{Y}_{2}-\mathrm{Y}_{1}\right) /\left(\mathrm{t}_{2}-\mathrm{t}_{1}\right)$, y la tasa de crecimiento relativo $(\mathrm{TCR}), \mathrm{TCR}=\left(\mathrm{Y}_{2}-\mathrm{Y}_{1} / \mathrm{Y}_{1}\right)\left(\mathrm{t}_{2}-\right.$ $\left.\mathrm{t}_{1}\right) \cdot 100$, donde, $\mathrm{Y}_{2}$ y $\mathrm{Y}_{1}=$ peso o longitud final e inicial, respectivamente, $\mathrm{t}_{2} \mathrm{y}_{1}=$ tiempo final e inicial de cultivo entre 2 muestreos (Wootton 1991).

Para obtener el índice de condición (IC, Walne \& Mann 1975), se utilizó el tejido suave y las conchas de 30 individuos seleccionados aleatoriamente en cada muestreo. El tejido suave fue secado en una estufa por $48 \mathrm{~h}$ a $80^{\circ} \mathrm{C}$ para obtener el peso seco. El IC fue calculado con la fórmula: $\mathrm{IC}=\mathrm{P} 1 \times 1000 / \mathrm{P} 2$, donde $\mathrm{P} 1$ es el peso seco del tejido suave y $\mathrm{P} 2$ es el peso seco de las conchas $(\mathrm{g})$.

Los datos fueron verificados para normalidad y homogeneidad de varianzas con las pruebas de Lilliefor y Bartlett, respectivamente (Sokal \& Rohlf 1995). Se aplicó un análisis de varianza (ANOVA) para los valores de crecimiento, mortalidad e IC empleando como factor el tiempo de cultivo. Cuando se detectaron diferencias significativas se aplicó una prueba a posteriori de Tukey $(\alpha=0,05)$. Para determinar el grado de asociación entre el crecimiento, supervivencia e IC con los parámetros fisicoquímicos, se realizó una correlación de Pearson (r), con su correspondiente prueba de significancia (Prueba t, $\alpha=0,05)$. Todos los estadísticos de realizaron con el Software Statistica 7.0 (Statsoft, Tulsa, OK) a un nivel de significancia de $P<0,05$.

\section{RESUltados}

Los valores mensuales de temperatura, $\mathrm{OD}$, salinidad y $\mathrm{pH}$ se muestran en la Figura 2. La temperatura promedio fue de 25,01 $\pm 5,29^{\circ} \mathrm{C}$, con una fluctuación de $17,5^{\circ} \mathrm{C}$ en diciembre, a $31,8^{\circ} \mathrm{C}$ registrados en septiembre. El OD (promedio $=6,10 \pm$ $\left.1,93 \mathrm{mg} \mathrm{L}^{-1}\right)$ mostró 2 picos, el más alto $\left(10,82 \mathrm{mg} \mathrm{L}^{-1}\right)$ en diciembre y el otro en septiembre $\left(9,55 \mathrm{mg} \mathrm{L}^{-1}\right)$. $\mathrm{La}$ concentración más baja de $\mathrm{OD}\left(2,57 \mathrm{mg} \mathrm{L}^{-1}\right)$ fue obtenida en octubre. La salinidad se mantuvo estable a lo largo del cultivo, variando de 30 a 37 . El pH promedio fue de 7,82 $\pm 0,39$, y fluctuó de 7,1 en enero a 8,6 en octubre. La profundidad mostró 2 registros bajos de $0,2 \mathrm{~m}$; uno en febrero y otro en octubre. La profundidad promedio fue de 1,66 $\pm 0,55 \mathrm{~m}$; con una variación de 0,2 m en febrero a $3 \mathrm{~m}$ en septiembre (Fig. 3).

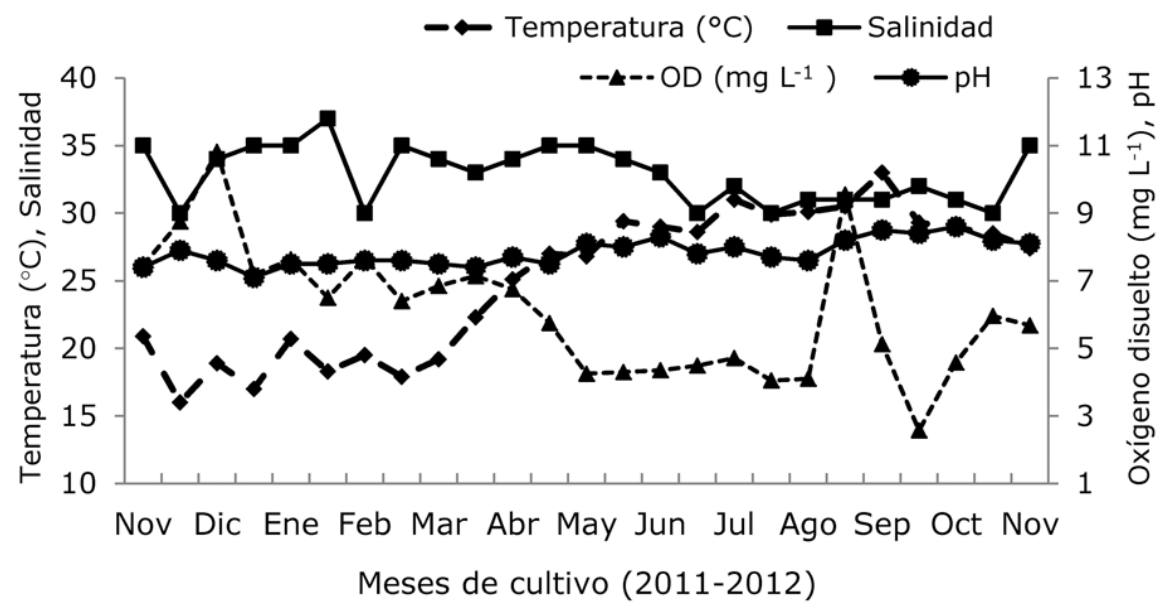

Figura 2. Temperatura, oxígeno disuelto, salinidad y pH del sitio de cultivo en el sistema lagunar Navachiste-M acapule, Sinaloa, M éxico / Temperature, dissolved oxygen, salinity and $\mathrm{pH}$ at the culture site in the Navachiste-Macapule lagoon system, Sinaloa, Mexico 


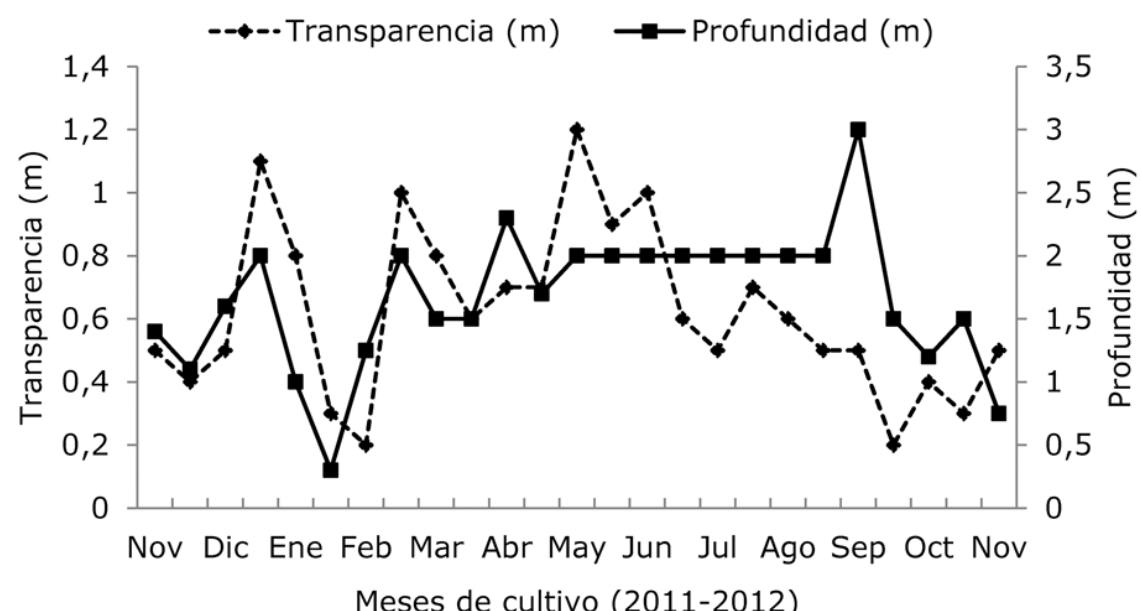

Figura 3. Transparencia y profundidad del sitio de cultivo en el sistema lagunar Navachiste-Macapule, Sinaloa, México / Transparency and depth at the culture site in the Navachiste-Macapule lagoon system, Sinaloa, Mexico

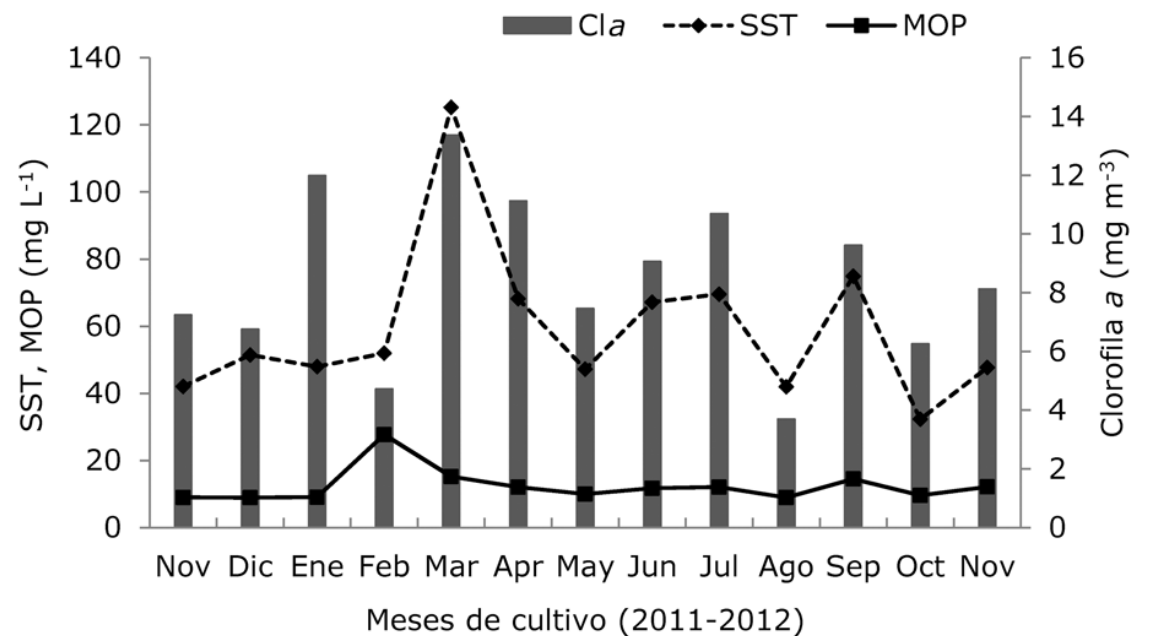

Figura 4. Sólidos suspendidos totales, materia orgánica particulada y clorofila a del sitio de cultivo en el sistema lagunar Navachiste-Macapule, Sinaloa, M éxico / Total suspended solids, particulate organic matter and chlorophyll $a$ at the culture site in the Navachiste-Macapule lagoon system, Sinaloa, Mexico

Las concentraciones de SST, MOP y Cl- $a$ mostraron tendencias similares entre febrero y marzo (Fig. 4). La concentración de SST presentó un rango desde 41,94 mg L-1 en agosto, hasta $125,22 \mathrm{mg} \mathrm{L}^{-1}$ en marzo, mientras que para MOP, el valor más bajo se observó en diciembre $\left(8,91 \mathrm{mg} \mathrm{L}^{-1}\right)$ y el más alto en marzo $\left(15,22 \mathrm{mg} \mathrm{L}^{-1}\right)$. En el caso de la $\mathrm{Cl}-a$, la mayor concentración $\left(13,36 \mathrm{mg} \mathrm{m}^{-3}\right)$ fue obtenida en marzo y la menor $\left(3,69 \mathrm{mg} \mathrm{m}^{-3}\right)$ en agosto.
La Tabla 1 muestra el análisis de varianza para las variables de altura, largo y ancho de las valvas y el peso total de $C$. gigas para cada mes de cultivo. Se observaron diferencias significativas $(P<0,05)$ para cada una de las variables.

Después de 12 meses de cultivo los organismos alcanzaron una talla promedio de 95,05 $\pm 9,21 \mathrm{~mm}$ de altura con los mayores incrementos en diciembre $(22,33 \mathrm{~mm})$ y febrero $(21,93$ 
$\mathrm{mm})$. El análisis de correlación mostró que la altura de las valvas se relacionó de manera positiva con el peso total $(\mathrm{r}=0,90 ; P=$ $0,0001)$, inversamente con la supervivencia $(\mathrm{r}=-0,65 ; P=$ $0,014)$, y no presentó relación con la concentración de Cl- $a$ $(\mathrm{r}=0,12 ; P=0,67)$. Para el peso total, se observó un incremento progresivo conforme crecía la altura de las valvas, hasta que en mayo y agosto se registraron disminuciones. El ostión fue sembrado con un peso inicial de $0,01 \mathrm{~g}$ en noviembre del 2011 y se cosechó con un peso final de 83,58 $\pm 17,18 \mathrm{~g}$ en noviembre 2012 (Fig. 5). Al igual que la altura de las valvas, el peso total

Tabla 1. Análisis de varianza para las variables altura, longitud y ancho de la concha, y peso total de C. gigas, cultivado en un sistema en suspensión en el sistema lagunar Navachiste-M acapule, Sinaloa, México ( $*$ diferencias significativas) / Analysis of variance for the total length and weight mean values of $C$. gigas cultured in suspension system at the Navachiste-Macapule lagoon system, Sinaloa, Mexico ( $*$ significant differences)

\begin{tabular}{ccrrcc}
\hline $\begin{array}{c}\text { Fuente de } \\
\text { variación }\end{array}$ & $\begin{array}{c}\text { Suma de } \\
\text { cuadrados }\end{array}$ & $\begin{array}{c}\text { Grados de } \\
\text { libertad }\end{array}$ & $\begin{array}{c}\text { Suma de } \\
\text { cuadrados } \\
\text { medios }\end{array}$ & F & $P$ \\
\hline Altura & 712.849 & 24 & 29.702 & 388,02 & $0,0001^{*}$ \\
Error & 93.771 & 1.225 & 76,54 & & \\
Longitud & 514.329 & 24 & $21.430,40$ & 196,40 & $0,0001^{*}$ \\
Error & 133.670 & 1.225 & 109,11 & & \\
Ancho & 102.529 & 24 & $4.272,05$ & 192,93 & $0,0001^{*}$ \\
Error & $27.124,5$ & 1.225 & 22,14 & & \\
Peso & 912.671 & 24 & 38.028 & 334,81 & $0,0001^{*}$ \\
Error & 139.135 & 1.225 & 113,57 & & \\
\hline
\end{tabular}

presentó una correlación inversa con la supervivencia $(\mathrm{r}=-0,87$; $P=0,0001)$ y positiva con la altura de las valvas $(\mathrm{r}=0,90 ; P=$ 0,0001), pero sin mostrar relación con la concentración de Cl$a(\mathrm{r}=0,02 ; P=0,94)$.

La tasa de crecimiento diaria del ostión fue de $0,25 \mathrm{~mm}$ día ${ }^{-1}$ para la altura de la concha y de $0,23 \mathrm{~g} \mathrm{día}^{-1}$ para el peso. En las Tablas 2 y 3 se observan los valores del crecimiento instantáneo (TCIR), absoluto (TCA) y relativo (TCR) para la altura de las valvas y el peso total de $C$. gigas. El máximo valor para las 3 tasas de crecimiento con relación a la altura de las valvas se registró en el mes de diciembre $(5,97 ; 0,74$ y $0,166 \mathrm{~mm} \mathrm{día}^{-1}$ para TCIR, TCA y TCR, respectivamente), mientras que valores negativos fueron detectados en mayo y agosto para los 3 indicadores. Los parámetros fisicoquímicos se relacionaron con cada una de las tasas de crecimiento. Para la temperatura, se observó una correlación inversa con las TCIR $(\mathrm{r}=-0,64 ; P=0,022)$ y TCA $(\mathrm{r}=-0,69 ; P=0,012)$, pero en el caso de la $\mathrm{Cl}-a$ no se observó correlación con ninguno de los indicadores de crecimiento $(\mathrm{r}=-0,09 ; P=0,76$ para TCIR; $\mathrm{r}=$ $-0,24 ; P=0,45$ para TCA, y r $=-0,16 ; P=0,60$ para TCR). El valor más alto para TCIR fue de $16,61 \mathrm{~mm} \mathrm{día}^{-1}$ y para el TCR de 4,83 $\mathrm{mm} \mathrm{día}^{-1}$ durante diciembre, y en coincidencia con la altura de las valvas, se observaron valores negativos de los 3 indicadores de crecimiento en mayo y agosto. Sólo se obtuvo una correlación inversa $(\mathrm{r}=-0,65 ; P=0,02)$ de la temperatura con el peso total de los ostiones (Tabla 4).

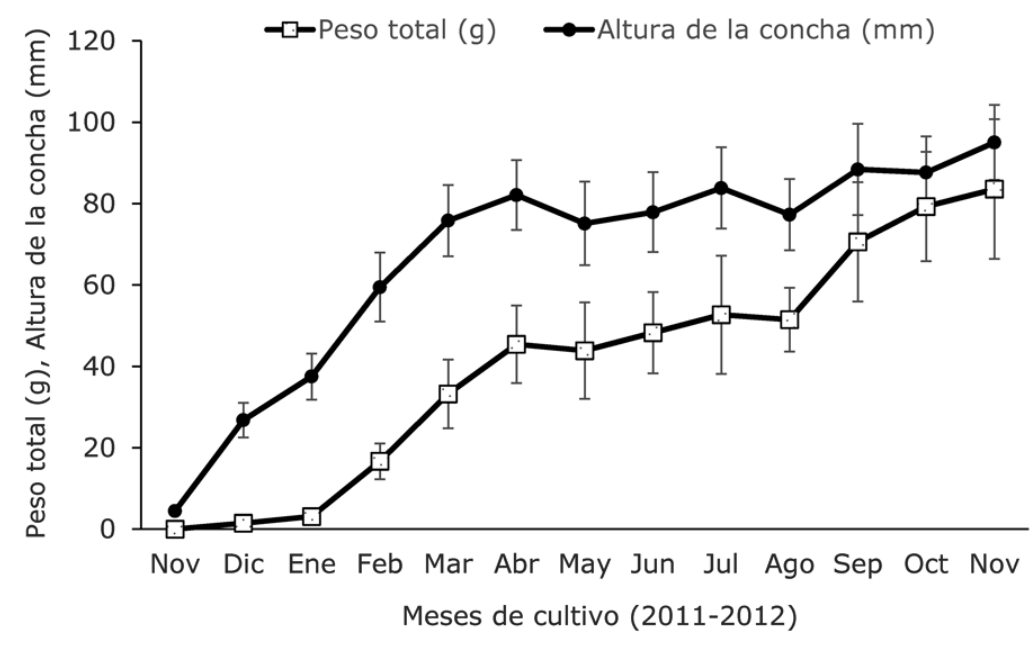

Figura 5. Altura de la concha y peso total mensual de $C$. gigas cultivado en el sistema lagunar Navachiste-M acapule, Sinaloa, México / Shell height and total weight of C. gigas cultured in the Navachiste-Macapule lagoon system, Sinaloa, Mexico 
Tabla 2. Indicadores de crecimiento para la altura de la concha ( $\mathrm{mm}$ $\mathrm{d}^{-1}$ ) de C. gigas cultivado en el sistema lagunar Navachiste-Macapule, Sinaloa, México. TasaAbs=Tasa de crecimiento absoluto, TasaRel=Tasa de crecimiento relativo, Tasalnst=Tasa de crecimiento instantáneo / Growth indexes for shell height $\left(\mathrm{mm} \mathrm{d}^{-1}\right)$ of $\mathrm{C}$. gigas cultured in the Navachiste-Macapule Lagoon system, Sinaloa, Mexico. TasaAbs= Absolute growth rate, TasaRel= Relative growth rate, Tasalnst= Instant growth rate

\begin{tabular}{lrrrr}
\hline \multicolumn{1}{c}{ Fecha } & $\begin{array}{c}\text { Altura } \\
(\mathrm{mm})\end{array}$ & TasaAbs & TasaRel & TasaInst \\
\hline Nov. 2011 & 4,46 & & & \\
Diciembre & 26,79 & 0,74 & 0,166 & 5,97 \\
Enero 2012 & 37,53 & 0,35 & 0,013 & 1,12 \\
Febrero & 59,46 & 0,73 & 0,019 & 1,53 \\
Marzo & 75,83 & 0,54 & 0,009 & 0,81 \\
Abril & 82,12 & 0,20 & 0,002 & 0,26 \\
Mayo & 75,12 & $-0,23$ & $-0,002$ & $-0,29$ \\
Junio & 77,89 & 0,09 & 0,001 & 0,12 \\
Julio & 83,84 & 0,19 & 0,002 & 0,24 \\
Agosto & 75,29 & $-0,28$ & $-0,003$ & $-0,35$ \\
Septiembre & 88,41 & 0,43 & 0,005 & 0,53 \\
Octubre & 87,66 & $-0,02$ & 0,0002 & $-0,02$ \\
Noviembre & 95,05 & 0,24 & 0,002 & 0,26 \\
\hline
\end{tabular}

Tabla 3. Indicadores de crecimiento para el peso total $\left(\mathrm{g} \mathrm{d}^{-1}\right)$ de $C$. gigas cultivado en el sistema lagunar Navachiste-M acapule, Sinaloa, México. TasaAbs= Tasa de crecimiento absoluto, TasaRel= Tasa de crecimiento relativo, Tasalnst= Tasa de crecimiento instantáneo / Growth indexes for total weight $\left(\mathrm{g} \mathrm{d}^{-1}\right)$ of $\mathrm{C}$. gigas cultured in the Navachiste-Macapule Lagoon system, Sinaloa, Mexico. TasaAbs= Absolute growth rate, Tasa Rel= Relative growth rate, Tasalnst= Instant growth rate

\begin{tabular}{lcccc}
\hline \multicolumn{1}{c}{ Fecha } & $\begin{array}{c}\text { Peso total } \\
(\mathrm{g})\end{array}$ & TasaAbs & TasaRel & TasaInst \\
\hline Nov. 2011 & 0,01 & & & \\
Diciembre & 1,46 & 0,04 & 4,833 & 16,61 \\
Enero 2012 & 3,10 & 0,05 & 0,037 & 2,50 \\
Febrero & 16,70 & 0,45 & 0,146 & 5,61 \\
Marzo & 33,25 & 0,55 & 0,033 & 2,29 \\
Abril & 45,44 & 0,40 & 0,012 & 1,04 \\
Mayo & 35,88 & $-0,31$ & $-0,007$ & $-0,78$ \\
Junio & 43,28 & 0,24 & 0,006 & 0,62 \\
Julio & 63,70 & 0,68 & 0,015 & 1,28 \\
Agosto & 49,18 & $-0,48$ & $-0,007$ & $-0,86$ \\
Septiembre & 70,61 & 0,71 & 0,014 & 1,20 \\
Octubre & 71,45 & 0,02 & 0,0003 & 0,03 \\
Noviembre & 83,58 & 0,40 & 0,005 & 0,52 \\
\hline
\end{tabular}

Tabla 4. Correlaciones de la temperatura y Cla con la altura de la concha y el peso total de $C$. gigas $(P<0,05 ;$ grados de libertad: 1,11), cultivado en suspensión en el sistema lagunar Navachiste-Macapule, Sinaloa, México / Correlations of temperature and $\mathrm{Cla}$ with the shell height and total weight of $\mathrm{C}$. gigas $(\mathrm{P}<0.05$; degrees of freedom: 1.11), cultured in suspended trays in the Navachiste-Macapule lagoon system, Sinaloa, Mexico

\begin{tabular}{lccccc}
\hline & $\mathrm{r}^{\dagger}$ & $\mathrm{a}$ & $\mathrm{b}$ & $\mathrm{F}$ & $P$ \\
\hline Altura de la concha/peso total & 0,96 & 32,14 & 0,87 & 47,27 & $0,0001^{*}$ \\
Altura de la concha/Cla & 0,12 & 56,54 & 1,22 & 0,18 & 0,67 \\
Altura de la concha/supervivencia & $-0,65$ & $1.034,7$ & $-9,83$ & 8,33 & $0,014^{*}$ \\
Peso total/Cla & 0,02 & 38,13 & 0,19 & 0,00 & 0,94 \\
Peso total/supervivencia & $-0,87$ & $1.366,62$ & $-13,47$ & 34,79 & $0,0001^{*}$ \\
\hline
\end{tabular}

$\mathrm{r}$ = Nivel de correlación; $\mathrm{a}=$ Ordenada al origen; $\mathrm{b}=$ pendiente. ${ }^{*}$ Correlación significativa

La supervivencia final (Fig. 6) fue de $94,72 \%$. El índice de condición fluctuó de 23,82 en febrero hasta 47,99 en noviembre 2012 (Fig. 7), con un promedio en el periodo de 34,65 $\pm 4,46$. La correlación canónica de las variables ambientales del sistema lagunar Navachiste-Macapule, Sinaloa, y los parámetros de crecimiento (altura de las valvas, peso total, mortalidad e IC) de $C$. gigas, se muestra en la Tabla 5. Las correlaciones entre la temperatura, y la altura de las valvas y el peso total del ostión presentaron los mayores valores $(r=0,7$ y $r=0,81$, respectivamente).

\section{DisCuSIón}

Hoy en día existe poca información sobre la tecnología de producción de la actividad ostrícola en el estado de Sinaloa, ya que la mayoría representan informes técnicos o reportes en foros locales (Nava et al. 1995, Góngora-Gómez et al. 2012), por lo que la realización y divulgación de este tipo de estudios, representan una prioridad a fin de establecer una estructura tecnológica para el desarrollo del cultivo comercial del ostión del Pacífico C. gigas en la zona. 


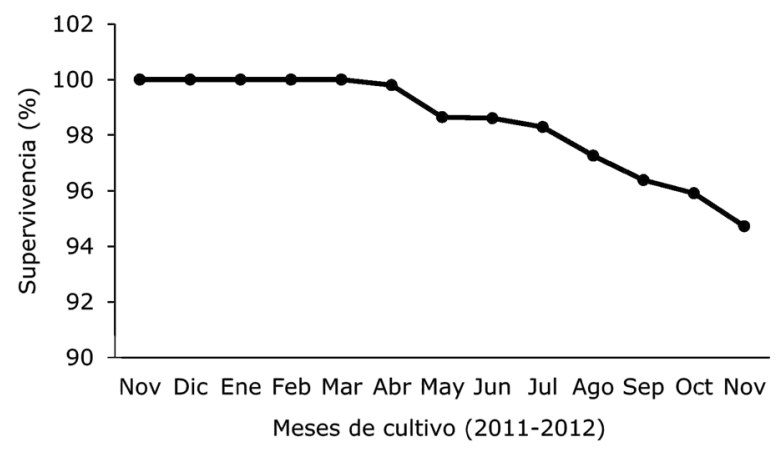

Figura 6. Supervivencia (\%) de C. gigas cultivado en el sistema lagunar Navachiste-Macapule, Sinaloa, México / Survival (\%) of C. gigas cultured in the Navachiste-Macapule Lagoon system, Sinaloa, México

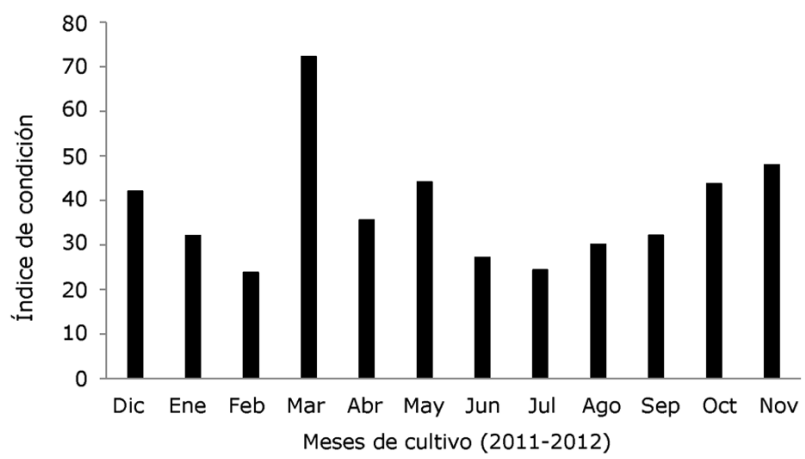

Figura 7. Índice de condición de C. gigas cultivado en suspensión en el sistema lagunar Navachiste-Macapule, Sinaloa, México / Condition index of $\mathrm{C}$. gigas cultured in suspended trays in the NavachisteMacapule lagoon system, Sinaloa, México

Tabla 5. Correlación canónica de las variables ambientales del sistema lagunar Navachiste-M acapule, Sinaloa, México, y las variables fisiológicas de C. gigas / Canonical correlation of environmental variables in the Navachiste-Macapule lagoon system, Sinaloa, Mexico, and physiological variables of C. gigas

\begin{tabular}{|c|c|c|c|c|c|c|c|c|}
\hline & $\mathrm{T}^{\circ}$ & Salinidad & $\mathrm{O}_{2}$ & $\mathrm{pH}$ & Transparencia & SST & MOP & $\mathrm{Cla}$ \\
\hline $\mathrm{AC}$ & $\mathbf{0 , 7 1 5 3 *}$ & $-0,3415$ & $-0,6205$ & 0,5010 & $-0,0586$ & 0,2245 & 0,1950 & 0,0359 \\
\hline PT & 0,8103 & $-0,4554$ & $-0,5863$ & 0,5863 & $-0,2317$ & $-0,0052$ & $-0,0298$ & $-0,1095$ \\
\hline $\mathrm{S}$ & 0,4408 & $-0,3308$ & $-0,0864$ & 0,1894 & $-0,1711$ & $-0,2797$ & $-0,2121$ & $-0,3215$ \\
\hline IC & $-0,2116$ & 0,5525 & 0,1810 & $-0,2726$ & 0,6163 & $-0,0156$ & 0,1991 & $-0,0545$ \\
\hline
\end{tabular}

$\mathrm{T}^{\circ}=$ Temperatura del agua; $\mathrm{O}_{2}=$ Oxígeno disuelto; $\mathrm{SST}=$ Sólidos suspendidos totales; $\mathrm{MOP}=$ Materia Orgánica Particulada; $\mathrm{Cl} a=$ Clorofila $a$

$\mathrm{AC}=$ Altura de la concha; $\mathrm{PT}=$ Peso total; $\mathrm{S}=$ Supervivencia; $\mathrm{IC}=$ Índice de condición

*Números en negritas $=$ correlación significativa

Los ostiones alcanzaron una altura de las valvas de 95,05 \pm 9,21 mm en 13 meses similares a los que reporta ChávezVillalba et al. (2007) en la Laguna El Soldado, del estado de Sonora donde $C$. gigas alcanzó un rango en la altura de las valvas de 97-118 mm en 13 meses de cultivo. Mientras que trabajando con diferentes densidades, Chávez-Villalba et al. (2010) determinaron tallas del ostión japonés de hasta 132,4 mm después de un año de engorda en Laguna La Cruz, Sonora. Pero estas diferencias en los resultados pueden explicarse por las condiciones ambientales en los diferentes sitios de cultivo, además de otros factores como el origen de la semilla, la talla de siembra y el método de cultivo.

La tasa de crecimiento de la concha de diversas especies de ostión tropical es mayor a la reportada para especies templadas y frías (Quayle \& Newkirk 1989). Por ejemplo, Kingsley-Smith et al. (2009) señalan que después de 23 meses de cultivo en diferentes localidades de la Bahía Chesapeake, Virginia, EUA, los ostiones C. virginica y C. ariakensis crecieron 0,053 y 0,090 mm día ${ }^{-1}$, valores que son bajos en comparación con los obtenidos en este trabajo $(0,25 \mathrm{~mm}$ día $^{-1}$ ) para $C$. gigas en 13 meses. Por otro lado, Castillo-Durán et al. (2010) reportaron tasas de crecimiento diaria de 0,098 y 0,250 mm día ${ }^{-1}$ para $C$. gigas y $C$. corteziensis, respectivamente, en verano, pero de 0,441 y 0,268 mm día ${ }^{-1}$ en invierno, lo cual explica la influencia que ejercen los factores del medio ambiente en diferentes latitudes y localidades, trabajando con diversas especies. El crecimiento y supervivencia de $C$. gigas en el presente estudio, mostraron que la especie es capaz de tolerar las variaciones ambientales que se registran en el sistema lagunar Navachiste-Macapule, donde la temperatura fue el factor de mayor influencia registrando una fluctuación de $17^{\circ} \mathrm{C}$. Considerando el presente resultado, se puede reafirmar que $C$. gigas es una especie que posee una alta tolerancia a cambios abruptos de temperatura, correspondiendo al marco ambiental de esteros y lagunas costeras en el Golfo de California (Osuna-López et al. 2009). 
De acuerdo a Fujiya (1970) y Cáceres-Martínez (1994), el intervalo óptimo de temperatura para el cultivo del ostión japonés es de $20-25^{\circ} \mathrm{C}$, pero en nuestro cultivo el organismo toleró temperaturas desde los 15 y a $\operatorname{los} 30^{\circ} \mathrm{C}$ sin afectar sus funciones alimenticias y reproductivas, lo que explica parcialmente los resultados obtenidos en el presente estudio. Otros factores como la salinidad y el pH se encontraron dentro del rango de crecimiento reportado para la especie que oscilan entre los 25-38 y 7-9, respectivamente (Korringa 1976).

Por ejemplo, el crecimiento (altura de las valvas y peso total) de este bivalvo no presentó correlación con la concentración de $\mathrm{Cl}-a$, lo que sugiere que su alimentación no depende exclusivamente de esa fuente de comida, sino de una combinación de Cl- $a$ y materia orgánica particulada, característica de hábitat con fondos lodosos (Sibaja 1985). También, Korringa (1976) menciona que el crecimiento del ostión está influenciado por las características del medio ambiente en combinación con el tipo y disponibilidad de alimento, factores que están fuertemente ligados a la latitud que se encuentre el sitio de cultivo (Roncarati et al. 2010). En las costas de Canadá y en el Mar del Norte donde se practica el cultivo intermareal, la tasa de crecimiento de los ostiones es de aproximadamente $5 \mathrm{~cm}$ por año, a pesar de la alta productividad primaria existente en esos lugares, mientras que en las costas del noroeste de México, lugar que en comparación presenta menor productividad, los animales pueden alcanzar una tasa de crecimiento de 18,5 cm anuales (Mazón-Suástegui 1996). En este caso, el crecimiento del organismo estuvo asociado a la disponibilidad de alimento, ya que el cultivo en suspensión mantiene permanentemente a los ostiones en el agua favoreciendo el índice de aclaramiento como el constante acceso al alimento a lo largo de la columna de agua que promueve mejores tasas de crecimiento en menor tiempo (Korringa 1976, Leighton 1979, Kang et al. 2003, Chávez-Villalba 2005, 2008; Betanzos-Vega et al. 2010).

Por otro lado, los valores de correlación obtenidos para los parámetros ambientales indican que la temperatura del agua ejerció un efecto mayor sobre la altura de la concha y el peso de $C$. gigas en el sistema lagunar Navachiste-Macapule, Sinaloa, lo cual coincide con lo reportado por Claudi \& Mackie (1994) para el crecimiento del mejillón cebra Dreissena polymorpha en poblaciones silvestres, pero contrario a lo encontrado por Buddensiek (1995) trabajando con la ostra Margaritifera margaritifera en cultivo. Las diferencias en resultados pueden ser parcialmente explicadas por las diversas especies estudiadas, la metodología y las condiciones ambientales o de cultivo.

El índice de condición es una medida muy útil para reconocer el estado nutritivo de los bivalvos debido al cambio temporal de las reservas alimenticias mismas que están influenciadas por la disponibilidad del alimento en conjunción con los factores ambientales (Soniat \& Ray 1985), y es ampliamente usado en C. gigas (Mason \& Nell 1995, Baghurst \& Mitchell 2002). El índice de condición se incrementó hasta mayo y disminuyó en junio y julio, mientras que el patrón de la $\mathrm{Cl}-a$ y MOP decreció a partir de mayo, lo cual sugiere que la disponibilidad de alimento pudiera haber afectado el ciclo reproductivo de este bivalvo y se haya presentado un evento de desove, lo cual es similar a lo reportado por Chávez-Villalba et al. (2007). Al mismo tiempo, la temperatura aumentó a más de $29^{\circ} \mathrm{C}$ pudiendo provocar que los ostiones aceleraran su actividad reproductiva. Es recomendable realizar estudios histológicos a lo largo del cultivo para determinar el desarrollo y madurez reproductiva del ostión.

La supervivencia de $C$. gigas durante todo el cultivo fue alta (> 94,72\%). Los factores que pueden afectar la supervivencia de bivalvos en cultivos suspendidos varían desde la presencia de epibiontes, depredación, competencia por espacio y alimento (Gallo-García et al. 2001, García-Ulloa et al. 2006), así como el efecto del continuo movimiento por las olas y corrientes (Serrano-Casillas 2004). En este trabajo, la temperatura y la disponibilidad de alimento no afectaron la supervivencia de los ostiones. La rutina de limpieza del equipo y animales cada 15 días puede explicar la baja mortalidad registrada, lo cual coincide con lo reportado por Gallo-García et al. (2001). La leve disminución en el porcentaje de supervivencia pudo ser debida a la presencia de juveniles de depredadores de la jaiba Callinectes sp., que fue encontrada esporádicamente dentro de las canastas (Sicard-González 1999).

Los resultados muestran que $C$. gigas alcanzó la talla comercial ( $80 \mathrm{~mm}$ de altura de las valvas) después de 8 meses de cultivo bajo las condiciones ambientales del sistema lagunar Navachiste-Macapule, Sinaloa, México, destacando: a) la obtención de una tasa de crecimiento diaria de $0,25 \mathrm{~mm}_{\text {día }}{ }^{-1}$ para la altura de la concha y de $0,23 \mathrm{~g} \mathrm{día}^{-1}$ para el peso, b) una supervivencia final mayor a $94 \%$, y c) la acción de la temperatura del agua en su crecimiento. Los datos obtenidos contribuyen con información básica necesaria para futuros estudios y ciclos de producción comercial de esta especie en la región.

\section{Agradecimientos}

Agradecemos al Instituto Politécnico Nacional (IPN) por el apoyo financiero y logístico a través de la Comisión de Operaciones y Fomento de Actividades Académicas (COFAAIPN), para la realización del proyecto, IPNSIP 20130858 (Estudio comparativo en el crecimiento y la supervivencia entre organismos diploides y triploides de ostión japonés $C$. gigas cultivados en el estero La Pitahaya, Guasave, Sinaloa). 


\section{LITERATURA CITADA}

APHA. 1995. Standard methods for the examination of water and wastewater: Multiple-Tube fermentation technique for members of the coliform group, $342 \mathrm{pp}$. American Public Health Association, Washington.

Baghurst BC \& JG Mitchell. 2002. Sex-specific growth and condition of the Pacific oyster (Crassostrea gigas Thunberg). Aquaculture Research 33: 1253-1263.

Betanzos-Vega A, C Siam-Lahera \& G Arencibia-Carballo. 2010. Variación de la salinidad y su relación con la distribución por talla del ostión de mangle, Villa Clara, Cuba. Revista Cubana de Investigaciones Pesqueras 27: 41-46.

Buddensiek V. 1995. The culture of juvenile freshwater pearl mussels Margaritifera margaritifera L. in cages: a contribution to conservation programmes and the knowledge of habitat requirements. Biology and Conservation 74: 3340.

Cáceres-Martínez C. 1994. Manual de ostricultura: Técnicas del engorda del ostión japonés (Crassostrea gigas), 46 pp. Universidad Autónoma de Baja California Sur, La Paz.

Cáceres-Martínez J, S Ramírez-Gutiérrez, R VázquezYeomans \& P Macías-Montes de Oca. 2004. Reproductive cycle and mortality of the Japanese oyster Crassostrea gigas cultured in Bahía Falsa, Baja California, México. Journal of Shellfish Research 23: 795-801.

Castillo-Durán A, J Chávez-Villalba, A Arreola-Lizárraga \& R Barraza-Guardado. 2010. Comparative growth, condition, and survival of juvenile Crassostrea gigas and $C$. corteziensis oysters cultivated in summer and winter. Ciencias Marinas 36(1): 29-39.

Chávez-Villalba J. 2014. Cultivo de ostión Crassostrea gigas: Análisis de 40 años de actividades en México. Hidrobiológica 24: $175-190$.

Chávez-Villalba J, MR López-Tapia, JM Mazón-Suástegui \& M Robles-Mungaray. 2005. Growth of the oyster Crassostrea corteziensis (Hertlein 1951) in Sonora, México. Aquaculture Research 36: 1337-1344.

Chávez-Villalba J, F Villelas-Ávila \& C Cáceres-Martínez. 2007. Reproduction, condition and mortality of the Pacific oyster Crassostrea gigas (Thunberg) along coastal Sonora, México. Aquaculture Research 38: 268-278.

Chávez-Villalba J, A Hernández-Ibarra, MR López-Tapia \& JM Mazón-Suástegui. 2008. Prospective culture of the Cortez oyster Crassostrea corteziensis from northwestern Mexico: Growth, gametogenic activity, and condition index. Journal Shellfish Research 27: 711-720.

Chávez-Villalba J, A Arreola-Lizárraga, S Burrola-Sánchez \& F Hoyos-Chairez. 2010. Growth, condition, and survival of the Pacific oyster Crassostrea gigas cultivated within and outside a subtropical lagoon. Aquaculture 300: 128-136.

Cheney DF, BF MacDonald \& RA Elston. 2000. Summer mortality of Pacific oysters, Crassostrea gigas (Thunberg): Initial findings of multiple environmental stressors in Puget Sound, Washington, 1998. Journal of Shellfish Research 19: 353-359.
Claudi R \& GL Mackie. 1994. Practical manual for zebra mussel monitoring and control, 227 pp. Lewis Publishers, CRC, Boca Raton.

Enríquez-Espinoza TL, JM Grijalva-Chon, R CastroLongoria \& J Ramos-Paredes. 2010. Perkinsus marinus in Crassostrea gigas from the Gulf of California. Diseases of Aquatic Organisms 89: 269-273.

Enríquez-Espinoza TL, R Castro-Longoria, F MendozaCano \& JM Grijalva-Chon. 2015. Perkinsus marinus in Crassostrea gigas and Chione fluctifraga from Kino Bay, Sonora, Mexico. Biotecnia 7(1): 10-13.

Fujiya M. 1970. Oyster farming in Japan. Helgoländer Wissenschaftliche Meeresunters 20: 464-479.

Gallo-García MC, M García-Ulloa, D Godínez-Siordia \& K Rivera-Gómez. 2001. Estudio preliminar sobre el crecimiento y supervivencia del ostión del Pacífico Crassostrea gigas (Thunberg, 1873) en Barra de Navidad, Jalisco, México. Universidad y Ciencia 17: 83-91.

García-Ulloa M, MC Gallo-García, OA González-Ochoa \& RA Chávez-Zazueta. 2006. Cultivo del ostión japonés Crassostrea gigas, en la costa media del Pacífico Mexicano. En: Jiménez-Quiroz MC \& E Espino-Barr (eds). Los recursos acuícolas y pesqueros de Jalisco, Colima y Michoacán, pp. 580-587. Instituto Nacional de la Pesca, SAGARPA, Centro Regional de Investigaciones Pesqueras de Manzanillo, Manzanillo.

Glude JB. 1974. A summary report of Pacific Coast oyster mortality investigations $1965-1972$, October $15-16^{\text {th }} 1974,28$ pp. Proceedings of the Third United States of America-Japan Meeting on Aquaculture, Tokyo.

Góngora-Gómez AM, M García-Ulloa, JA HernándezSepúlveda \& AL Domínguez-Orozco. 2012. Crecimiento del ostión Crassostrea gigas (Thunberg, 1795) cultivado en el estero La Piedra, Sinaloa, México. Avances en Investigación Agropecuaria 16(2): 91-104.

Grabowski JH, CH Peterson, SP Powers, D Gaskill \& HC Summerson. 2004. Growth and survivorship of non-native (Crassostrea gigas and Crassostrea ariakensis) versus native oysters (Crassostrea virginica). Journal of Shellfish Research 23: 781-793.

Helm MM. 2015. Cultured aquatic species information programme. Crassostrea gigas (Thunberg, 1793). FAO, Fisheries and Aquaculture Department, Rome. <http:// www.fao.org/fishery/culturedspecies/Crassostrea_gigas/en >

Kang C, M Park, P Lee, W Choi \& W Lee. 2003. Seasonal variations in conditions, reproductive activity, and biochemical composition of the Pacific oyster, Crassostrea gigas (Thunberg), in suspended culture in two coastal bays of Korea. Journal of Shellfish Research 19: 771-778.

Kingsley-Smith PR, HD Darwell, ML Kellogg, SM Allen, SK Allen Jr, DW Meritt, KT Paytner Jr \& MW Luckenbach. 2009. Survival and growth of triploid Crassostrea virginica (Gmelin, 1791) and C. ariakensis (Fujita, 1913) in bottom environments of Chesapeake Bay: Implications for an introduction. Journal of Shellfish Research 28(2): 169-184. 
Korringa P. 1976. Farming the cupped oyster of the genus Crassostrea, 224 pp. Elsevier, Amsterdam.

Leighton DL. 1979. A growth prolific for the rock scallop Hinnites multirugosuls held at several depths off La Jolla, California. Marine Biology 51: 229-232.

Maeda-Martínez AN. 2008. Estado actual del cultivo de bivalvos en México. In: Lovatelli A, A Farias \& I Uriarte (eds). Taller regional de la FAO sobre el estado actual del cultivo y manejo de moluscos bivalvos y su proyección futura: Factores que afectan su sustentabilidad en América Latina. Actas de Pesca de la FAO 12: 91-100.

Mason CJ \& JA Nell. 1995. Condition index and chemical composition of meats of Sydney Rock oysters (Saccostrea commercialis) and Pacific oyster (Crassostrea gigas) at four sites in Port Stephens, NSW. Marine and Freshwater Research 46: 873-881.

Mazón-Suástegui JM. 1996. Cultivo del ostión japonés Crassostrea gigas. En: Casas V \& DG Ponce (eds). Estudio del potencial pesquero y acuícola de Baja California Sur 1: 625-650. México.

Nava HJH, H Nava \& R Ramos. 1995. Cultivo experimental de crecimiento y engorda del ostión japonés en cajas ostreófilas en el estero de Teacapán, Sinaloa. Gaceta Informativa, Unidad de Educación en Ciencia y Tecnología del Mar, SEP. 13: 20-22.

Osuna-López JI, MG Frías-Espiricueta, G López-López, HM Zazueta-Padilla, G Izaguirre-Fierro, F Páez-Osuna, AC Ruíz-Fernández \& D Voltolina. 2009. $\mathrm{Cd}, \mathrm{Pb}$ and organochloride pesticides of Mytella strigata (Pelecypoda: Mytillidae) of six coastal lagoons of NW Mexico. Boletín de Investigaciones Marinas y Costeras 38(2): 233-239.

Quayle DB \& GF Newkirk. 1989. Farming bivalve mollusks: Methods for study and development. Advances in World Aquaculture 1: 1-294. The World Aquaculture Society Los Angeles.

Ren JS, AH Ross \& DR Schiel. 2000. Functional description of feeding and energetics of the Pacific oyster Crassostrea gigas in New Zealand. Marine Ecology Progress Series 208: 119-130.

Roncarati A, A Fellici, A Dess, F Leila \& M Paolo. 2010. Trails on Pacific oyster (Crassostrea gigas Thunberg) reared in the middle Adriatic Sea by means of different trays. Aquaculture International 18: 35-43.

Royer J, M Ropert \& K Costil. 2007. Spatio-temporal changes in mortality, growth and condition of the Pacific oyster, Crassostrea gigas, in Normandy (France). Journal of Shellfish Research 26: 973-984.

Serrano-Casillas G. 2004. Crecimiento y supervivencia de la almeja catarina Argopecten ventricosus (Sowerby II, 1842), bajo condiciones de cultivo en suspensión y fondo en Bahía Concepción, Baja California Sur, México. Tesis de Maestría, Baja California Sur, México, 164 pp.
Sibaja WG. 1985. Dimensiones de la concha del mejillón Mytella strigata Hanley (Bivalvia: Mytillidae), de la Playa de Lepanto, Puntarenas, Costa Rica. Revista de Biología Tropical 33(1): 59-60.

Sicard-González MT. 1999. Temperatura letal superior y temperatura óptima de una población de almeja catarina (Argopecten ventricosus, Sowerby II, 1842). Tesis de Maestría, Instituto Politécnico Nacional, La Paz, 99 pp.

Sokal RR \& FJ Rohlf. 1995. Biometry: the principles and practice of statistics in biological research, $887 \mathrm{pp}$. WH Freeman, New York.

Soletchnick P, O Le Moine, N Faury, D Razet, P Geairon \& P Goulletquer. 1999. Mortalité de l'huitre Crassostrea gigas dans le bassin de Marennes-Oléron: Étude de la variabilité spatiale de son environnement et de sa biologie par un système d'informations géographiques (SIG). Aquatic Living Resources 12:131-143.

Soniat TM \& SM Ray. 1985. Relationships between possible available food and the composition, condition and reproductive state of oysters from Galveston Bay, Texas. Contributions in Marine Science 28: 109-121.

Strickland JDH \& TR Parsons. 1972. A practical handbook of seawater analysis. Fisheries Research Board of Canada, Bulletin 167: 1-310.

Ulloa PA, A Martínez \& D Escobedo. 2003. Fitoplancton del litoral del municipio de Guasave, Sinaloa. Resúmenes. IX Congreso de la Asociación de Investigadores del mar de Cortés, A. C. y III Simposium Internacional del Mar de Cortés, La Paz, Baja California Sur, Mayo 5-9, pp. 95-96.

Vázquez-Yeomans R, M García-Ortega \& J CáceresMartínez. 2010. Gill erosion and herpesvirus in Crassostrea gigas cultured in Baja California, México. Diseases of Aquatic Organisms 89: 137-144.

Villanueva-Fonseca LC \& CM Escobedo-Bonilla. 2013. Prevalencia del protozoario Perkinsus sp. en un cultivo de ostión japonés Crassostrea gigas en Sinaloa, México. Latin American Journal of Aquatic Research 41: 996-1002.

Walne PR \& R Mann. 1975. Growth and biochemical composition in Ostrea edulis and Crassostrea gigas. In: Barnes H (ed). $9^{\text {th }}$ European Marine Biology Symposium, pp. 587-607. Aberdeen University Press, Scotland.

Wootton RF. 1991. Ecology of teleost fishes. Fish and fisheries, 404 pp. Series I. Chapman \& Hall, London.

Xiao J, S Ford, F Zhang \& X Guo. 2005. Studies on mass summer mortality of cultured zhikong scallops (Chlamys farrei Jones et Preston) in China. Aquaculture 250: 602615. 